\title{
On a new type of degenerate poly-Fubini numbers and polynomials
}

\author{
Waseem A. Khan \\ Department of Mathematics and Natural Sciences, Prince Mohammad Bin Fahd \\ University, P.O Box 1664, Al Khobar 31952, Saudi Arabia \\ E-mail: wkhan1@pmu.edu.sa
}

\begin{abstract}
In this paper, we introduce a new type of degenerate poly-Fubini polynomials and numbers, are called degenerate poly-Fubini polynomials and numbers, by using the degenerate polylogarithm function and derive several properties on the degenerate poly-Fubini polynomials and numbers. In the last section, we also consider the degenerate unipoly-Fubini polynomials attached to an arithmetic function, by using the degenerate polylogarithm function and investigate some identities of those polynomials. In particular, we give some new explicit expressions and identities of degenerate unipoly polynomials related to special numbers and polynomials.
\end{abstract}

2010 Mathematics Subject Classification.: 11B73, 11B83, 11S80, 05A19.

Keywords: Degenerate polylogarithm functions, degenerate poly-Fubini polynomials, degenerate unipoly functions, degenerate unipoly-Fubini polynomials.

\section{Introduction}

Carlitz's [2, 3] initiated a study of degenerate versions of some special polynomials and numbers, namely the degenerate Bernoulli and Euler polynomials and numbers. Kim and Kim et al. [14-28] have studied the degenerate versions of special numbers and polynomials actively. This idea provides a powerful tool to define special numbers and polynomials of their degenerate versions. We can say that the notion of degenerate version forms a special class of polynomials because of their great applicability. The most important applications of these polynomials are in the theory of finite differences, analytic number theory, application in classical analysis, and statistics. Despite the applicability of special functions in classical analysis and statistics, they also arise in communications systems, quantum mechanics, nonlinear wave propagation, electric circuit theory, electromagnetic theory, etc.

It is well known, that the two-variable Fubini polynomials are defined by means of the following generating function (see [29])

$$
\frac{e^{x t}}{1-y\left(e^{t}-1\right)}=\sum_{n=0}^{\infty} F_{n}(x, y) \frac{t^{n}}{n !} .
$$

When $x=0$ in (1.1), the two variable Fubini polynomials $F_{n}(x, y)$ reduce to the usual Fubini polynomials given by

$$
\frac{1}{1-y\left(e^{t}-1\right)}=\sum_{n=0}^{\infty} F_{n}(y) \frac{t^{n}}{n !},(\text { see }[1,4,7]) \text {. }
$$

It is easy to see that

$$
F_{n}\left(x,-\frac{1}{2}\right)=E_{n}(x), F_{n}\left(-\frac{1}{2}\right)=E_{n}
$$

and

$$
F_{n}(y)=\sum_{k=0}^{n} S_{2}(n, k) k ! y^{k}
$$


For $y=1$ in (1.2), we get the familiar Fubini numbers $F_{n}(1)=F_{n}$ as follows

$$
\frac{1}{\left.2-e^{t}\right)}=\sum_{n=0}^{\infty} F_{n} \frac{t^{n}}{n !},(\text { see }[37]) .
$$

For more information about the applications of the usual Fubini polynomials and numbers (see $[5,11,12,34,35])$. by

For any non-zero $\lambda \in \mathbb{R}$ (or $\mathbb{C}$ ), the degenerate exponential function is defined

$$
e_{\lambda}^{x}(t)=(1+\lambda t)^{\frac{x}{\lambda}}, e_{\lambda}(t)=(1+\lambda t)^{\frac{1}{\lambda}},(\text { see }[9,13]) .
$$

By binomial expansion, we get

$$
e_{\lambda}^{x}(t)=\sum_{n=0}^{\infty}(x)_{n, \lambda} \frac{t^{n}}{n !},(\text { see }[19,20,25]),
$$

where $(x)_{0, \lambda}=1,(x)_{n, \lambda}=(x-\lambda)(x-2 \lambda) \cdots(x-(n-1) \lambda),(n \geq 1)$.

Note that

$$
\lim _{\lambda \longrightarrow 0} e_{\lambda}^{x}(t)=\sum_{n=0}^{\infty} x^{n} \frac{t^{n}}{n !}=e^{x t}
$$

In $[2,3]$ Carlitz introduced the degenerate Bernoulli and degenerate Euler polynomials are defined by

$$
\frac{z}{e_{\lambda}(z)-1} e_{\lambda}^{x}(z)=\sum_{j=0}^{\infty} B_{j, \lambda}(x) \frac{z^{j}}{j !}, \frac{2}{e_{\lambda}(z)+1} e_{\lambda}^{x}(z)=\sum_{j=0}^{\infty} E_{j, \lambda}(x) \frac{z^{j}}{j !},
$$

respectively.

In the case when $x=0, B_{j, \lambda}=B_{j, \lambda}(0)$ are called the degenerate Bernoulli numbers and $x=0, E_{j, \lambda}=E_{j, \lambda}(0)$ are called the degenerate Euler numbers.

Obviously

$$
\lim _{\lambda \longrightarrow 0} \beta_{n}(x ; \lambda)=B_{n}(x), \lim _{\lambda \longrightarrow 0} E_{n}(x ; \lambda)=E_{n}(x) .
$$

For $s \in \mathbb{Z}$, the polylogaritm function is defined by a power series in $\mathrm{z}$ as

$$
\operatorname{Li}_{s}(z)=\sum_{j=1}^{\infty} \frac{j^{n}}{j^{s}}=z+\frac{z^{2}}{2^{s}}+\frac{z^{3}}{3^{s}},(|z|<1),(\text { see, }[8,32]) .
$$

It is notice that

$$
\operatorname{Li}_{1}(z)=\sum_{j=1}^{\infty} \frac{z^{j}}{j}=-\log (1-z) .
$$

For $\lambda \in \mathbb{R}$, Kim-Kim [26] defined the degenerate version of the logarithm function, denoted by $\log _{\lambda}(1+z)$ as follows:

$$
\log _{\lambda}(1+z)=\sum_{j=1}^{\infty} \lambda^{j-1}(1)_{j, 1 / \lambda} \frac{z^{j}}{j !},(\text { see, }[11])
$$

being the inverse of the degenerate version of the exponential function $e_{\lambda}(z)$ as has been shown below

$$
e_{\lambda}\left(\log _{\lambda}(z)\right)=\log _{\lambda}\left(e_{\lambda}(z)\right)=z
$$


It is noteworthy to mention that

$$
\lim _{\lambda \rightarrow 0} \log _{\lambda}(1+z)=\sum_{j=1}^{\infty}(-1)^{j-1} \frac{z^{j}}{j !}=\log (1+z) .
$$

The degenerate polylogarithm function [26] is defined by Kim-Kim to be

$$
l_{k, \lambda}(z)=\sum_{j=1}^{\infty} \frac{(-\lambda)^{j-1}(1)_{j, 1 / \lambda}}{(j-1) ! j^{k}} z^{n},(k \in \mathbb{Z},|z|<1) .
$$

It is clear that

$$
\lim _{\lambda \rightarrow 0} l_{k, \lambda}(z)=\sum_{j=1}^{\infty} \frac{z^{j}}{j^{k}}=\operatorname{Li}_{k}(z),(\text { see }[3,4]) .
$$

From (1.10) and (1.11), we get

$$
l_{1, \lambda}(z)=\sum_{j=1}^{\infty} \frac{(-\lambda)^{j-1}(1)_{j, 1 / \lambda}}{j !} z^{j}=-\log _{\lambda}(1-z) .
$$

Very recently, Kim-Kim [26] introduced the new type degenerate version of the Bernoulli polynomials and numbers, by using the degenerate polylogarithm function as follows

$$
\frac{l_{k, \lambda}\left(1-e_{\lambda}(-z)\right)}{1-e_{\lambda}(-z)} e_{\lambda}^{u}(z)=\sum_{j=0}^{\infty} \beta_{j, \lambda}^{(k)}(u) \frac{z^{j}}{j !} .
$$
bers.

On setting $x=0, \beta_{j, \lambda}^{(k)}=\beta_{j, \lambda}^{(k)}(0)$ are called the degenerate poly-Bernoulli num-

The two variable degenerate Fubini polynomials $F_{n, \lambda}(x ; y)$ are defined by

$$
\begin{aligned}
& \frac{1}{1-y\left((1+\lambda t)^{\frac{1}{\lambda}}-1\right)}(1+\lambda t)^{\frac{x}{\lambda}} \\
& =\sum_{n=0}^{\infty} F_{n, \lambda}(x ; y) \frac{t^{n}}{n !},(\text { see }[24]) .
\end{aligned}
$$
bers.

When $x=0$ and $y=1, F_{n, \lambda}=F_{n, \lambda}(0 ; 1)$ are called the degenerate Fubini num-

Lee-Kim-Jang [33] introduced the type 2 degenerate poly-Euler polynomials and numbers as follows

$$
\frac{\operatorname{Ei}_{k}(\log (1+2 t))}{t\left(e_{\lambda}(t)+1\right)} e_{\lambda}^{x}(t)=\sum_{n=0}^{\infty} E_{n, \lambda}^{(k)}(x) \frac{t^{n}}{n !} .
$$
bers.

When $x=0, E_{n, \lambda}^{(k)}=E_{n, \lambda}^{(k)}(0)$ are called the type 2 degenerate poly-Euler num-

The degenerate Stirling numbers of the first kind [18] are defined by

$$
\frac{1}{k !}\left(\log _{\lambda}(1+z)\right)^{k}=\sum_{j=k}^{\infty} S_{1, \lambda}(j, k) \frac{z^{j}}{j !},(k \geq 0),(\text { see }[1-15]) .
$$

It is clear that

$$
\lim _{\lambda \rightarrow 0} S_{1, \lambda}(j, k)=S_{1}(j, k),
$$


are calling the Stirling numbers of the first kind given by

$$
\frac{1}{k !}(\log (1+z))^{k}=\sum_{j=k}^{\infty} S_{1}(j, k) \frac{z^{j}}{j !},(k \geq 0),(\text { see }[8-12]) .
$$

The degenerate Stirling numbers of the second kind [16] are given by

$$
\frac{1}{k !}\left(e_{\lambda}(z)-1\right)^{k}=\sum_{j=k}^{\infty} S_{2, \lambda}(j, k) \frac{z^{j}}{j !},(k \geq 0) .
$$

Note here that

$$
\lim _{\lambda \rightarrow 0} S_{2, \lambda}(j, k)=S_{2}(j, k),
$$

standing for the Stirling numbers of the second kind given by means of the following generating function:

$$
\frac{1}{k !}\left(e^{z}-1\right)^{k}=\sum_{j=k}^{\infty} S_{2}(j, k) \frac{z^{j}}{j !},(k \geq 0),(\text { see, }[1-37]) .
$$

In this paper, we construct the new type of degenerate poly-Fubini polynomials and numbers by using degenerate polylogarithm function and derive several properties on the degenerate poly-Fubini numbers and polynomials. Furthermore, we introduce the degenerate unipoly-Fubini polynomials attached to an arithmetic function, by using degenerate polylogarithm function and investigate some properties for those polynomials. Also, we give some new explicit expressions and identities of degenerate unipoly polynomials related to special numbers and polynomials.

\section{A new type of degenerate poly-Fubini numbers and polynomials}

In this section, we define the new type of degenerate poly-Fubini numbers and polynomials by using the degenerate polylogarithm function which are called the new type of degenerate poly-Fubini numbers and polynomials and investigate some properties of these polynomials. We start following definition as follows.

Now, we consider the new type of 2 variable degenerate poly-Fubini polynomials which are called the new type of 2 variable degenerate poly-Fubini polynomials and given by

$$
\frac{l_{k, \lambda}\left(1-e_{\lambda}(-t)\right)}{t\left(1-y\left((1+\lambda t)^{\frac{1}{\lambda}}-1\right)\right)}(1+\lambda t)^{\frac{x}{\lambda}}=\sum_{n=0}^{\infty} F_{n, \lambda}^{(k)}(x ; y) \frac{t^{n}}{n !},(k \in \mathbb{Z}) .
$$

At the point $F_{n, \lambda}^{(k)}(1)=F_{n, \lambda}^{(k)}(0 ; 1)$ are called the new type of degenerate polyFubini numbers, where $l_{k, \lambda}\left(1-e_{\lambda}(-t)\right)=-\log _{\lambda}\left(1-1+e_{\lambda}(-t)\right)=t$.

Note that

$$
\begin{aligned}
& \sum_{n=0}^{\infty} F_{n, \lambda}^{(1)}(x ; y y) \frac{t^{n}}{n !}=\frac{l_{1, \lambda}\left(1-e_{\lambda}(-t)\right)}{t\left(1-y\left((1+\lambda t)^{\frac{1}{\lambda}}-1\right)\right)}(1+\lambda t)^{\frac{x}{\lambda}} \\
& =\frac{1}{1-y\left((1+\lambda t)^{\frac{1}{\lambda}}-1\right)}(1+\lambda t)^{\frac{x}{\lambda}}=\sum_{n=0}^{\infty} F_{n, \lambda}(x ; y) \frac{t^{n}}{n !} .
\end{aligned}
$$

Obviously, we have

$$
F_{n, \lambda}^{(1)}(x ; y)=F_{n, \lambda}(x ; y),(n \geq 0)
$$


From (2.1), we see that

$$
\begin{aligned}
\sum_{n=0}^{\infty} F_{n, \lambda}^{(k)} & (x ; y) \frac{z^{n}}{n !}=\frac{l_{k, \lambda}\left(1-e_{\lambda}(-t)\right)}{t\left(1-y\left((1+\lambda t)^{\frac{1}{\lambda}}-1\right)\right)}(1+\lambda t)^{\frac{x}{\lambda}} \\
& =\left(\sum_{n=0}^{\infty} F_{n, \lambda}^{(k)}(y) \frac{t^{n}}{n !}\right)\left(\sum_{m=0}^{\infty}(x)_{\lambda, m} \frac{t^{m}}{m !}\right) \\
& =\left(\sum_{n=0}^{\infty} F_{n, \lambda}^{(k)}(y) \frac{t^{n}}{n !}\right)\left(\sum_{m=0}^{\infty}(x)_{\lambda, m} \frac{t^{m}}{m !}\right) \\
& =\sum_{n=0}^{\infty}\left(\sum_{m=0}^{n}\left(\begin{array}{c}
n \\
m
\end{array}\right) F_{n-m, \lambda}^{(k)}(y)(x)_{\lambda, m}\right) \frac{z^{n}}{n !}
\end{aligned}
$$

Therefore, by equation (2.3), we obtain the following theorem.

Theorem 2.1. Let $n \geq 0$, we have

$$
F_{n, \lambda}^{(k)}(x ; y)=\sum_{m=0}^{n}\left(\begin{array}{c}
n \\
m
\end{array}\right) F_{n-m, \lambda}^{(k)}(y)(x)_{\lambda, m} .
$$

From (), we observe that

$$
\begin{gathered}
\frac{d}{d x} l_{k, \lambda}\left(1-e_{\lambda}(-x)\right)=\frac{d}{d x} \sum_{m=1}^{\infty} \frac{(-\lambda)^{n-1}(1)_{m, 1 / \lambda}}{(m-1) ! m^{k}}\left(1-e_{\lambda}(-x)\right)^{m} \\
\frac{1}{\left(1-e_{\lambda}(-x)\right)} l_{k-1, \lambda}\left(1-e_{\lambda}(-x)\right) .
\end{gathered}
$$

From (2.1) and (2.4), for $k \geq 2$, we obtain

$$
\begin{gathered}
\sum_{n=0}^{\infty} F_{n, \lambda}^{(k)}(y) \frac{x^{n}}{n !}=\frac{1}{x\left(1-y\left((1+\lambda x)^{\frac{1}{\lambda}}-1\right)\right)} l_{k-1, \lambda}\left(1-e_{\lambda}(-x)\right) \\
=\frac{1}{x\left(1-y\left((1+\lambda x)^{\frac{1}{\lambda}}-1\right)\right)} \int_{0}^{x} \frac{e_{\lambda}^{1-\lambda}(-t)}{1-e_{\lambda}(-t)} \int_{0}^{t} \frac{e_{\lambda}^{1-\lambda}(-t)}{1-e_{\lambda}(-t)} \cdots \int_{0}^{t} \frac{e_{\lambda}^{1-\lambda}(-t)}{1-e_{\lambda}(-t)} t d t d t \cdots d t \\
=\frac{1}{x\left(1-y\left((1+\lambda x)^{\frac{1}{\lambda}}-1\right)\right)} \int_{0}^{x} \underbrace{\frac{e_{\lambda}^{1-\lambda}(-t)}{1-e_{\lambda}(-t)} \int_{0}^{t} \frac{e_{\lambda}^{1-\lambda}(-t)}{1-e_{\lambda}(-t)} \cdots \int_{0}^{t} \frac{e_{\lambda}^{1-\lambda}(-t)}{1-e_{\lambda}(-t)}}_{(k-2)-\text { times }} t d t d t \cdots d t .
\end{gathered}
$$

Therefore, by (2.5), we get the following theorem.

Theorem 2.2. Let $k \geq 2$. Then

$$
\begin{gathered}
\sum_{n=0}^{\infty} F_{n, \lambda}^{(k)}(y) \frac{x^{n}}{n !} \\
=\frac{1}{x\left(1-y\left((1+\lambda x)^{\frac{1}{\lambda}}-1\right)\right)} \int_{0}^{x} \underbrace{\frac{e_{\lambda}^{1-\lambda}(-t)}{1-e_{\lambda}(-t)} \int_{0}^{t} \frac{e_{\lambda}^{1-\lambda}(-t)}{1-e_{\lambda}(-t)} \cdots \int_{0}^{t} \frac{e_{\lambda}^{1-\lambda}(-t)}{1-e_{\lambda}(-t)}}_{(k-2)-\text { times }} t d t d t \cdots d t .
\end{gathered}
$$

For $k=2$, by Theorem 2.2, we get

$$
\sum_{n=0}^{\infty} F_{n, \lambda}^{(2)}(y) \frac{x^{n}}{n !}=\frac{1}{x\left(1-y\left((1+\lambda x)^{\frac{1}{\lambda}}-1\right)\right)} \int_{0}^{x} \frac{-t}{e_{\lambda}(-t)-1} e_{\lambda}^{1-\lambda}(-t) d t
$$




$$
\begin{gathered}
=\frac{1}{x\left(1-y\left((1+\lambda x)^{\frac{1}{\lambda}}-1\right)\right)} \int_{0}^{x} \sum_{n=0}^{\infty} B_{n, \lambda}(1-\lambda) \frac{(-t)^{n}}{n !} d t \\
=\frac{1}{1-y\left((1+\lambda x)^{\frac{1}{\lambda}}-1\right)} \sum_{m=0}^{\infty}(-1)^{m} \frac{B_{m, \lambda}(1-\lambda)}{m+1} \frac{x^{m}}{m !} \\
\quad=\left(\sum_{n=0}^{\infty} F_{n, \lambda}(y) \frac{x^{n}}{n !}\right)\left(\sum_{m=0}^{\infty}(-1)^{m} \frac{B_{m, \lambda}(1-\lambda)}{m+1} \frac{x^{m}}{m !}\right) \\
\text { L.H.S }=\sum_{n=0}^{\infty}\left(\sum_{m=0}^{n}\left(\begin{array}{c}
n \\
m
\end{array}\right)(-1)^{m} \frac{B_{m, \lambda}(1-\lambda)}{m+1} F_{n-m, \lambda}(y)\right) \frac{x^{n}}{n !} .
\end{gathered}
$$

Therefore, by (2.6), we get the following theorem.

Theorem 2.3. Let $n \geq 0$, we have

$$
F_{n, \lambda}^{(2)}(y)=\sum_{m=0}^{n}\left(\begin{array}{c}
n \\
m
\end{array}\right)(-1)^{m} \frac{B_{m, \lambda}(1-\lambda)}{m+1} F_{n-m, \lambda}(y)=\sum_{m=0}^{n}\left(\begin{array}{c}
n \\
m
\end{array}\right)(-1)^{n-m} \frac{B_{n-m, \lambda}(1-\lambda)}{n-m+1} F_{m, \lambda}(y) .
$$

In general, from (2.8), we note that

$$
\begin{aligned}
& \sum_{n=0}^{\infty} F_{n, \lambda}^{(k)}(y) \frac{x^{n}}{n !}=\frac{1}{x\left(1-y\left((1+\lambda x)^{\frac{1}{\lambda}}-1\right)\right)} \int_{0}^{x} \underbrace{\frac{e_{\lambda}^{1-\lambda}(-t)}{1-e_{\lambda}(-t)} \int_{0}^{t} \frac{e_{\lambda}^{1-\lambda}(-t)}{1-e_{\lambda}(-t)} \cdots \int_{0}^{t} \frac{e_{\lambda}^{1-\lambda}(-t)}{1-e_{\lambda}(-t)}}_{(k-2)-\text { times }} t d d t \cdots d t \\
& =\frac{1}{x\left(1-y\left((1+\lambda x)^{\frac{1}{\lambda}}-1\right)\right)} \sum_{n_{1}, n_{2}, \cdots, n_{k-1}=m}^{\infty}(-1)^{m}\left(\begin{array}{l}
n \\
n_{1}, n_{2}, \cdots, n_{k}
\end{array}\right) \\
& \times \frac{B_{n_{1}, \lambda}}{n_{1}+1} \frac{B_{n_{2}, \lambda}}{n_{1}+n_{2}+1} \cdots \frac{B_{n_{k-1}, \lambda}}{n_{1}+\cdots+n_{k-1}+1} \\
& \text { L.H.S }=\sum_{n=0}^{\infty}\left(\sum_{n_{1}, n_{2}, \cdots, n_{k}=m}\left(\begin{array}{l}
n \\
m
\end{array}\right)(-1)^{m}\left(\begin{array}{l}
n \\
n_{1}, n_{2}, \cdots, n_{k}
\end{array}\right)\right. \\
& \left.\times \frac{B_{n_{1}, \lambda}}{n_{1}+1} \frac{B_{n_{2}, \lambda}}{n_{1}+n_{2}+1} \cdots \frac{B_{n_{k-1}, \lambda}}{n_{1}+\cdots+n_{k-1}+1} F_{n-m, \lambda}(y)\right) \frac{x^{n}}{n !} .
\end{aligned}
$$

Therefore by comparing the coefficients on both sides of (2.7), we obtain the following theorem.

Theorem 2.4. Let $k \in \mathbb{Z}$ and $n \geq 0$, we have

$$
\begin{gathered}
F_{n, \lambda}^{(k)}(y)=\sum_{n_{1}, n_{2}, \cdots, n_{k}=m}\left(\begin{array}{c}
n \\
m
\end{array}\right)(-1)^{m}\left(\begin{array}{l}
n \\
n_{1}, n_{2}, \cdots, n_{k}
\end{array}\right) \frac{B_{n_{1}, \lambda}}{n_{1}+1} \frac{B_{n_{2}, \lambda}}{n_{1}+n_{2}+1} \\
\times \cdots \frac{B_{n_{k-1}, \lambda}}{n_{1}+\cdots+n_{k-1}+1} F_{n-m, \lambda}(y) .
\end{gathered}
$$

From (2.1), we note that

$$
\begin{gathered}
\frac{l_{k, \lambda}\left(1-e_{\lambda}(-t)\right)}{t}=\left(1-y\left((1+\lambda t)^{\frac{1}{\lambda}}-1\right)\right) \sum_{n=0}^{\infty} F_{n, \lambda}^{(k)}(y) \frac{t^{n}}{n !} \\
=\sum_{n=0}^{\infty} F_{n, \lambda}^{(k)}(y) \frac{t^{n}}{n !}-y \sum_{n=0}^{\infty} \sum_{m=0}^{n}\left(\begin{array}{c}
n \\
m
\end{array}\right)(1)_{m, \lambda} F_{n-m, \lambda}^{(k)}(y) \frac{t^{n}}{n !}+y \sum_{n=0}^{\infty} F_{n, \lambda}^{(k)}(y) \frac{t^{n}}{n !}
\end{gathered}
$$




$$
=(1+y) \sum_{n=0}^{\infty} F_{n, \lambda}^{(k)}(y) \frac{t^{n}}{n !}-y \sum_{n=0}^{\infty} \sum_{m=0}^{n}\left(\begin{array}{c}
n \\
m
\end{array}\right)(1)_{m, \lambda} F_{n-m, \lambda}^{(k)}(y) \frac{t^{n}}{n !} .
$$

On the other hand,

$$
\begin{gathered}
\frac{l_{k, \lambda}\left(1-e_{\lambda}(-t)\right)}{t}=\frac{1}{t} \sum_{m=1}^{\infty} \frac{(1)_{m, 1 / \lambda}(-\lambda)^{m-1}}{(m-1) ! m^{k}}\left(1-e_{\lambda}(-t)\right)^{m} \\
=\frac{1}{t} \sum_{m=0}^{\infty} \frac{(1)_{m+1,1 / \lambda}(-\lambda)^{m-1}}{m !(m+1)^{k}}\left(1-e_{\lambda}(-t)\right)^{m+1} \frac{(m+1) !}{(m+1) !} \\
=\frac{1}{t} \sum_{m=0}^{\infty} \frac{(1)_{m+1,1 / \lambda}(-\lambda)^{m-1}}{(m+1)^{k-1}} \sum_{n=m+1}^{\infty} S_{2, \lambda}(n, m+1)(-1)^{n-m-1} \frac{t^{n}}{n !} \\
\text { L.H.S }=\sum_{n=0}^{\infty}\left(\sum_{m=0}^{n} \frac{(1)_{m+1,1 / \lambda}(\lambda)^{m-1}}{(m+1)^{k-1}} \frac{S_{2, \lambda}(n+1, m+1)(-1)^{n-1}}{n+1}\right) \frac{t^{n}}{n !} .
\end{gathered}
$$

Therefore, by (2.8) and (2.9), we obtain the following theorem.

Theorem 2.5. Let $k \in \mathbb{Z}$ and $n \geq 0$, we have

$$
\begin{gathered}
F_{n, \lambda}^{(k)}(y)=\frac{1}{1+y}\left[y \sum_{m=0}^{n}\left(\begin{array}{c}
n \\
m
\end{array}\right)(1)_{m, \lambda} F_{n-m, \lambda}^{(k)}(y)\right. \\
\left.+\sum_{m=0}^{n} \frac{(1)_{m+1,1 / \lambda}(\lambda)^{m-1}}{(m+1)^{k-1}} \frac{S_{2, \lambda}(n+1, m+1)(-1)^{n-1}}{n+1}\right] .
\end{gathered}
$$

For $k=1$ in Theorem 2.5., we get the following corollary

Corollary 2.3. For $n \geq 0$, we have

$$
\begin{gathered}
F_{n, \lambda}(y)=\frac{1}{1+y}\left[y \sum_{m=0}^{n}\left(\begin{array}{c}
n \\
m
\end{array}\right)(1)_{m, \lambda} F_{n-m, \lambda}(y)\right. \\
\left.+\sum_{m=0}^{n} \frac{(1)_{m+1,1 / \lambda}(\lambda)^{m-1} S_{2, \lambda}(n+1, m+1)(-1)^{n-1}}{n+1}\right] .
\end{gathered}
$$

From (2.1), we note that

$$
\begin{gathered}
\sum_{n=0}^{\infty}\left(F_{n, \lambda}^{(k)}(x+1 ; y)-F_{n, \lambda}^{(k)}(x ; y)\right) \frac{z^{n}}{n !} \\
=\frac{l_{k, \lambda}\left(1-e_{\lambda}(-t)\right)}{t\left(1-y\left((1+\lambda t)^{\frac{1}{\lambda}}-1\right)\right)}(1+\lambda t)^{\frac{x}{\lambda}}\left((1+\lambda t)^{\frac{1}{\lambda}}-1\right) \\
=\frac{l_{k, \lambda}\left(1-e_{\lambda}(-t)\right)}{t}\left(\frac{1}{y}\left(\left(\frac{(1+\lambda t)^{\frac{x}{\lambda}}}{1-y\left((1+\lambda t)^{\frac{1}{\lambda}}-1\right)}\right)-(1+\lambda t)^{\frac{x}{\lambda}}\right)\right) \\
=\frac{1}{y}\left(\frac{l_{k, \lambda}\left(1-e_{\lambda}(-t)\right)(1+\lambda t)^{\frac{x}{\lambda}}}{t\left(1-y\left((1+\lambda t)^{\frac{1}{\lambda}}-1\right)\right)}-\frac{l_{k, \lambda}\left(1-e_{\lambda}(-t)\right)(1+\lambda t)^{\frac{x}{\lambda}}}{t}\right) \\
=\frac{1}{y}\left(\sum_{n=0}^{\infty} F_{n, \lambda}^{(k)}(x ; y) \frac{t^{n}}{n !}-\sum_{n=0}^{\infty}\left(\sum_{m=0}^{n} \frac{(1)_{m+1,1 / \lambda}(\lambda)^{m-1}}{(m+1)^{k-1}} \frac{S_{2, \lambda}(n+1, m+1)(-1)^{n-1}}{n+1}\right) \frac{t^{n}}{n !} \sum_{n=0}^{\infty}(x)_{n, \lambda} \frac{t^{n}}{n !}\right) \\
=\frac{1}{y} \sum_{n=0}^{\infty}\left(F_{n, \lambda}^{(k)}(x ; y)-\sum_{l=0}^{n} \sum_{m=0}^{l}\left(\begin{array}{l}
n \\
l
\end{array}\right) \frac{S_{1, \lambda}(l+1, m+1)}{(m+1)^{k-1}}(x)_{n-l, \lambda}\right) \frac{t^{n}}{n !} .
\end{gathered}
$$


By comparing the coefficients on both sides of (2.19), we get the following theorem.

Theorem 2.6. Let $k \in \mathbb{Z}$ and $n \geq 0$, we have $y F_{n, \lambda}^{(k)}(x+1 ; y)=(y+1) F_{n, \lambda}^{(k)}(x ; y)-\sum_{l=0}^{n} \sum_{m=0}^{l}\left(\begin{array}{l}n \\ l\end{array}\right) \frac{1}{(m+1)^{k-1}} \frac{S_{1, \lambda}(l+1, m+1)}{l+1}(x)_{n-l, \lambda}$.

When $x=0$ and $x=-1$ in Theorem (2.6), we get

$$
y F_{n, \lambda}^{(k)}(1 ; y)=(y+1) F_{n, \lambda}^{(k)}(y)-\sum_{l=0}^{n} \sum_{m=0}^{l}\left(\begin{array}{l}
n \\
l
\end{array}\right) \frac{1}{(m+1)^{k-1}} \frac{S_{1, \lambda}(l+1, m+1)}{l+1},(n \geq 0) .
$$

and

$$
y F_{n, \lambda}^{(k)}(y)=(y+1) F_{n, \lambda}^{(k)}(-1 ; y)-\sum_{l=0}^{n} \sum_{m=0}^{l}\left(\begin{array}{l}
n \\
l
\end{array}\right) \frac{1}{(m+1)^{k-1}} \frac{S_{1, \lambda}(l+1, m+1)}{l+1}(-1)_{n-l, \lambda},(n \geq 0) .
$$

Now, we observe that

$$
\begin{gathered}
\left(\frac{l_{k, \lambda}\left(1-e_{\lambda}(-t)\right)}{t\left(1-y_{1}\left((1+\lambda t)^{\frac{1}{\lambda}}-1\right)\right)}(1+\lambda t)^{\frac{x_{1}}{\lambda}}\right)\left(\frac{l_{k, \lambda}\left(1-e_{\lambda}(-t)\right)}{t\left(1-y_{2}\left((1+\lambda t)^{\frac{1}{\lambda}}-1\right)\right)}(1+\lambda t)^{\frac{x_{2}}{\lambda}}\right) \\
=\frac{\operatorname{Ei}_{k, \lambda}\left(\log _{\lambda}(1+t)\right)}{t}\left(\frac{y_{2}}{y_{2}-y_{1}} \frac{(1+\lambda t)^{\frac{x_{1}+x_{2}}{\lambda}}}{1-y_{2}\left((1+\lambda t)^{\frac{1}{\lambda}}-1\right)}-\frac{y_{1}}{y_{2}-y_{1}} \frac{(1+\lambda t)^{\frac{x_{1}+x_{2}}{\lambda}}}{1-y_{2}\left((1+\lambda t)^{\frac{1}{\lambda}}-1\right)}\right) \\
=\sum_{n=0}^{\infty}\left(\frac{y_{2} F_{n, \lambda}^{(k)}\left(x_{1}+x_{2} ; y_{2}\right)-y_{1} F_{n, \lambda}^{(k)}\left(x_{1}+x_{2} ; y_{1}\right)}{y_{2}-y_{1}}\right) \frac{t^{n}}{n !} .
\end{gathered}
$$

On the other hand,

$$
\begin{gathered}
\left(\frac{l_{k, \lambda}\left(1-e_{\lambda}(-t)\right)}{t\left(1-y_{1}\left((1+\lambda t)^{\frac{1}{\lambda}}-1\right)\right)}(1+\lambda t)^{\frac{x_{1}}{\lambda}}\right)\left(\frac{l_{k, \lambda}\left(1-e_{\lambda}(-t)\right)}{t\left(1-y_{2}\left((1+\lambda t)^{\frac{1}{\lambda}}-1\right)\right)}(1+\lambda t)^{\frac{x_{2}}{\lambda}}\right) \\
=\left(\sum_{n=0}^{\infty} F_{n, \lambda}^{(k)}\left(x_{1} ; y_{1}\right) \frac{t^{n}}{n !}\right)\left(\sum_{m=0}^{\infty} F_{m, \lambda}^{(k)}\left(x_{2} ; y_{2}\right) \frac{t^{m}}{m !}\right) \\
=\sum_{n=0}^{\infty}\left(\sum_{m=0}^{n}\left(\begin{array}{c}
n \\
m
\end{array}\right) F_{n-m, \lambda}^{(k)}\left(x_{1} ; y_{1}\right) F_{m, \lambda}^{(k)}\left(x_{2} ; y_{2}\right)\right) \frac{t^{n}}{n !} .
\end{gathered}
$$

Therefore, by (2.20) and (2.21), we get the following theorem.

Theorem 2.7. Let $k \in \mathbb{Z}$ and $n \geq 0$, we have

$$
\begin{aligned}
& \sum_{m=0}^{n}\left(\begin{array}{c}
n \\
m
\end{array}\right) F_{n-m, \lambda}^{(k)}\left(x_{1} ; y_{1}\right) F_{m, \lambda}^{(k)}\left(x_{2} ; y_{2}\right) \\
= & \frac{y_{2} F_{n, \lambda}^{(k)}\left(x_{1}+x_{2} ; y_{2}\right)-y_{1} F_{n, \lambda}^{(k)}\left(x_{1}+x_{2} ; y_{1}\right)}{y_{2}-y_{1}} .
\end{aligned}
$$

For $x_{1}=x_{2}=0$ in Theorem 2.7, we have

Corollary 2.4. Let $k \in \mathbb{Z}$ and $n \geq 0$, we have

$$
\sum_{m=0}^{n}\left(\begin{array}{c}
n \\
m
\end{array}\right) F_{n-m, \lambda}^{(k)}\left(y_{1}\right) F_{m, \lambda}^{(k)}\left(y_{2}\right)=\frac{y_{2} F_{n, \lambda}^{(k)}\left(y_{2}\right)-y_{1} F_{n, \lambda}^{(k)}\left(y_{1}\right)}{y_{2}-y_{1}}
$$


From (2.1), we note that

$$
\begin{gathered}
\sum_{n=0}^{\infty} F_{n, \lambda}^{(k)}(x ; y-1) \frac{t^{n}}{n !}=\frac{l_{k, \lambda}\left(1-e_{\lambda}(-t)\right)}{t\left(1-(y-1)\left((1+\lambda t)^{\frac{1}{\lambda}}-1\right)\right)}(1+\lambda t)^{\frac{x}{\lambda}} \\
=\frac{l_{k, \lambda}\left(1-e_{\lambda}(-t)\right)(1+\lambda t)^{\frac{x}{\lambda}}}{t\left(1-y\left((1+\lambda t)^{\frac{1}{\lambda}}-1\right)+(1+\lambda t)^{\frac{1}{\lambda}}-1\right)} \\
=\frac{l_{k, \lambda}\left(1-e_{\lambda}(-t)\right)(1+\lambda t)^{\frac{x-1}{\lambda}}}{t\left(1-y(1-\lambda t)^{\frac{1}{\lambda}}\right)} \\
=\frac{l_{k, \lambda}\left(1-e_{\lambda}(-t)\right)(1-\lambda(-t))^{-\frac{1-x}{\lambda}}}{t\left(1+y\left((1-\lambda(-t))^{-\frac{1}{\lambda}}-1\right)\right)} \\
=\sum_{n=0}^{\infty} F_{n,-\lambda}^{(k)}(1-x ;-y) \frac{(-1)^{n} t^{n}}{n !}
\end{gathered}
$$
orem.

Comparing the coefficients on both sides of (2.22), we obtain the following the-

Theorem 2.8. Let $k \in \mathbb{Z}$ and $n \geq 0$, we have

$$
F_{n, \lambda}^{(k)}(x ; y-1)=(-1)^{n} F_{n,-\lambda}^{(k)}(1-x ;-y) .
$$

On setting $x=0$ in Theorem 2.8 , we get

Corollary 2.5. Let $k \in \mathbb{Z}$ and $n \geq 0$, we have

$$
F_{n, \lambda}^{(k)}(y-1)=(-1)^{n} F_{n,-\lambda}^{(k)}(1 ;-y) .
$$

From (2.1), we see that

$$
\begin{gathered}
\sum_{n=0}^{\infty} F_{n, \lambda}^{(k)}(y) \frac{t^{n}}{n !}=\frac{l_{k, \lambda}\left(1-e_{\lambda}(-t)\right)}{t\left(1-y\left((1+\lambda t)^{\frac{1}{\lambda}}-1\right)\right)} \\
=\left(\frac{l_{k, \lambda}\left(1-e_{\lambda}(-t)\right)}{t}\right)\left(\sum_{r=0}^{\infty} y^{r}\left((1+\lambda t)^{\frac{1}{\lambda}}-1\right)^{r}\right) \\
=\frac{1}{t}\left(\sum_{m=1}^{\infty} \frac{(-\lambda)^{m-1}(1)_{m, 1 / \lambda}\left(1-e_{\lambda}(-t)\right)^{m}}{(m-1) ! m^{k}}\right)\left(\sum_{n=0}^{\infty} \sum_{r=0}^{n} y^{r} S_{2, \lambda}(n, r) \frac{t^{n}}{n !}\right) \\
=\left(\sum_{l=0}^{\infty} \sum_{m=0}^{l} \frac{1}{(m+1)^{k-1}} \frac{(-\lambda)^{m}(1)_{m+1,1 / \lambda}(-1)^{l-1} S_{2, \lambda}(l+1, m+1)}{l+1} \frac{t^{l}}{l !}\right)\left(\sum_{n=0}^{\infty} \sum_{r=0}^{n} y^{r} S_{2, \lambda}(n, r) \frac{t^{n}}{n !}\right) \\
=\sum_{n=0}^{\infty}\left(\sum_{l=0}^{n} \sum_{r=0}^{n-l} \sum_{m=0}^{l}\left(\begin{array}{c}
n \\
l
\end{array}\right) \frac{1}{(m+1)^{k-1}} \frac{(1)_{m+1, \lambda} S_{1, \lambda}(l+1, m+1)}{l+1} y^{r} S_{2, \lambda}(n-l, r)\right) \frac{t^{n}}{n !} .
\end{gathered}
$$

Therefore, by (2.23), we get the following theorem.

Theorem 2.9. Let $k \in \mathbb{Z}$ and $n \geq 0$, we have

$$
F_{n, \lambda}^{(k)}(y)=\sum_{l=0}^{n} \sum_{r=0}^{n-l} \sum_{m=0}^{l}\left(\begin{array}{l}
n \\
l
\end{array}\right) \frac{1}{(m+1)^{k-1}} \frac{(1)_{m+1, \lambda} S_{1, \lambda}(l+1, m+1)}{l+1} y^{r} S_{2, \lambda}(n-l, r) .
$$

Corollary 2.6. Let $k \in \mathbb{Z}$ and $n \geq 0$, we have 


$$
F_{n, \lambda}(y)=\sum_{l=0}^{n} \sum_{r=0}^{n-l} \sum_{m=0}^{l}\left(\begin{array}{l}
n \\
l
\end{array}\right) \frac{(1)_{m+1, \lambda} S_{1, \lambda}(l+1, m+1)}{l+1} y^{r} S_{2, \lambda}(n-l, r) .
$$

\section{New type degenerate unipoly-Fubini numbers and polynomials}

In this section, we introduce new type of degenerate unipoly-Fubini polynomials by arising from degenerate polylogarithm function and derive several properties of these polynomials.

Let $p$ be any arithmetic function which is a real or complex valued function defined on the set of positive integers $\mathbb{N}$. Kim-Kim [15] defined the unipoly function attached to polynomials $p(x)$ by

$$
u_{k}(x \mid p)=\sum_{n=1}^{\infty} \frac{p(n)}{n^{k}} x^{n},(k \in \mathbb{Z}) .
$$

Moreover,

$$
u_{k}(x \mid 1)=\sum_{n=1}^{\infty} \frac{x^{n}}{n^{k}}=\operatorname{Li}_{k}(x),(\text { see }[8]),
$$

is the ordinary polylogaritm function.

In this paper, we define the degenerate unipoly function attached to polynomials $p(x)$ as follows:

$$
u_{k, \lambda}(x \mid p)=\sum_{i=1}^{\infty} p(i) \frac{(-\lambda)^{i-1}(1)_{i, 1 / \lambda}}{i^{k}} x^{i} .
$$

It is worthy to note that

$$
u_{k, \lambda}\left(x \mid \frac{1}{\Gamma}\right)=l_{k, \lambda}(x)
$$

is the degenerate polylogarithm function.

Now, we define the new type of degenerate unipoly-Fubini polynomials attached to polynomials $p(x)$ by

$$
\frac{u_{k, \lambda}\left(1-e_{\lambda}(-t) \mid p\right)}{t\left(1-y\left((1+\lambda t)^{\frac{1}{\lambda}}-1\right)\right)}(1+\lambda t)^{\frac{x}{\lambda}}=\sum_{n=0}^{\infty} F_{n, \lambda, p}^{(k)}(x) \frac{t^{n}}{n !} .
$$

In the case when $x=0, F_{n, \lambda, p}^{(k)}=F_{n, \lambda, p}^{(k)}(0)$ are called the new type degenerate unipoly-Fubini numbers attached to $p$.

From (3.5), we see

$$
\begin{aligned}
& \sum_{n=0}^{\infty} F_{n, \lambda, \frac{1}{\Gamma}}^{(k)} \frac{t^{n}}{n !}=\frac{1}{t\left(1-y\left((1+\lambda t)^{\frac{1}{\lambda}}-1\right)\right)} u_{k, \lambda}\left(1-e_{\lambda}(-t) \mid \frac{1}{\Gamma}\right) \\
& =\frac{1}{t\left(1-y\left((1+\lambda t)^{\frac{1}{\lambda}}-1\right)\right)} \sum_{r=1}^{\infty} \frac{(-\lambda)^{r-1}(1)_{r, 1 / \lambda}\left(1-e_{\lambda}(-t)\right)^{r}}{r^{k}(r-1) !} \\
& =\frac{1}{t\left(1-y\left((1+\lambda t)^{\frac{1}{\lambda}}-1\right)\right)} l_{k, \lambda}\left(1-e_{\lambda}(-t)\right)=\sum_{n=0}^{\infty} F_{n, \lambda}^{(k)} \frac{t^{n}}{n !} .
\end{aligned}
$$

Thus, by (3.6), we have 


$$
F_{n, \lambda, \frac{1}{\Gamma}}^{(k)}=F_{n, \lambda}^{(k)}
$$

From (3.5), we have

$$
\begin{gathered}
\sum_{n=0}^{\infty} F_{n, \lambda, p}^{(k)} \frac{t^{n}}{n !}=\frac{1}{t\left(1-y\left((1+\lambda t)^{\frac{1}{\lambda}}-1\right)\right)} u_{k, \lambda}\left(1-e_{\lambda}(-t) \mid p\right) \\
=\frac{1}{t\left(1-y\left((1+\lambda t)^{\frac{1}{\lambda}}-1\right)\right)} \sum_{m=1}^{\infty} \frac{p(m)(-\lambda)^{m-1}(1)_{m, 1 / \lambda}}{m^{k}}\left(1-e_{\lambda}(-t)\right)^{m} \\
=\left(\frac{1}{t\left(1-y\left((1+\lambda t)^{\frac{1}{\lambda}}-1\right)\right)}\right) \sum_{l=1}^{\infty}\left(\sum_{m=1}^{\infty} \frac{m ! p(m)(-1)^{l-1} \lambda^{m-1}(1)_{m, 1 / \lambda}}{m^{k}}\right) S_{2, \lambda}(l, m) \frac{t^{l}}{l !} \\
=\left(\sum_{n=0}^{\infty} F_{n, \lambda} \frac{t^{n}}{n !}\right)\left(\sum_{l=0}^{\infty}\left(\sum_{m=1}^{l+1} \frac{m ! p(m)(-1)^{l} \lambda^{m-1}(1)_{m, 1 / \lambda}}{m^{k}} \frac{S_{2, \lambda}(l+1, m)}{l+1}\right)\right) \frac{t^{l}}{l !} \\
\text { L.H.S }=\sum_{n=0}^{\infty}\left(\sum_{l=0}^{n} \sum_{m=1}^{l+1}\left(\begin{array}{l}
n \\
l
\end{array}\right) \frac{m ! p(m)(-1)^{l} \lambda^{m-1}(1)_{m, 1 / \lambda}}{m^{k}} \frac{S_{2, \lambda}(l+1, m)}{l+1} F_{n-l, \lambda}\right) \frac{t^{n}}{n !} .
\end{gathered}
$$

By equation (3.8), we get the following theorem.

Theorem 3.1. Let $n \geq 0$ and $k \in \mathbb{Z}$. Then

$$
F_{n, \lambda, p}^{(k)}=\sum_{l=0}^{n} \sum_{m=1}^{l+1}\left(\begin{array}{l}
n \\
l
\end{array}\right) \frac{m ! p(m)(-1)^{l} \lambda^{m-1}(1)_{m, 1 / \lambda}}{m^{k}} \frac{S_{2, \lambda}(l+1, m)}{l+1} F_{n-l, \lambda} .
$$

In particular,

$$
F_{n, \lambda, \frac{1}{\Gamma}}^{(k)}(y)=F_{n, \lambda}^{(k)}(y)=\sum_{l=0}^{n} \sum_{m=1}^{l+1}\left(\begin{array}{l}
n \\
l
\end{array}\right) \frac{m !(-1)^{l} \lambda^{m-1}(1)_{m, 1 / \lambda}}{m^{k}} \frac{S_{2, \lambda}(l+1, m)}{l+1} F_{n-l, \lambda} .
$$

From (3.5), we observe that

$$
\begin{gathered}
\sum_{n=0}^{\infty} F_{n, \lambda}^{(k, p)}(x ; y) \frac{t^{n}}{n !}=\frac{u_{k, \lambda}\left(\left(1-e_{\lambda}(-t)\right) \mid p\right)}{t\left(1-y\left((1+\lambda t)^{\frac{1}{\lambda}}-1\right)\right)}\left(e_{\lambda}^{-1}(t)-1+1\right)^{x} \\
=\frac{u_{k, \lambda}\left(\left(1-e_{\lambda}(-t)\right) \mid p\right)}{t\left(1-y\left((1+\lambda t)^{\frac{1}{\lambda}}-1\right)\right)} \sum_{m=0}^{\infty}\left(\begin{array}{c}
x+m-1 \\
m
\end{array}\right)\left(1-e_{\lambda}^{-1}(t)\right)^{m} \\
=\left(\sum_{n=0}^{\infty} F_{n, \lambda, p}^{(k)}(y) \frac{t^{n}}{n !}\right)\left(\sum_{m=0}^{\infty}(x)^{(m)} \sum_{l=m}^{\infty} S_{2, \lambda}(l, m ;-m) \frac{t^{l}}{l !}\right) \\
\quad=\left(\sum_{n=0}^{\infty} F_{n, \lambda, p}^{(k)}(y) \frac{t^{n}}{n !}\right)\left(\sum_{l=0}^{\infty} \sum_{m=0}^{l}(x)^{(m)} S_{2, \lambda}(l, m ;-m) \frac{t^{l}}{l !}\right) \\
\text { L.H.S }=\sum_{n=0}^{\infty}\left(\sum_{l=0}^{n} \sum_{m=0}^{l}\left(\begin{array}{c}
n \\
l
\end{array}\right) F_{n-l, \lambda, p}^{(k)}(y)(x)^{(m)} S_{2, \lambda}(l, m ;-m)\right) \frac{t^{n}}{n !} .
\end{gathered}
$$

From (3.11), we obtain the following theorem.

Theorem 3.2. Let $n \geq 0$ and $k \in \mathbb{Z}$. Then we have 


$$
F_{n, \lambda, p}^{(k)}(x ; y)=\sum_{l=0}^{n} \sum_{m=0}^{l}\left(\begin{array}{l}
n \\
l
\end{array}\right) F_{n-l, \lambda, p}^{(k)}(y)(x)^{(m)} S_{2, \lambda}(l, m ;-m) .
$$

From (3.5), we have

$$
\begin{aligned}
& \sum_{n=0}^{\infty} F_{n, \lambda, p}^{(k)}(x) \frac{t^{n}}{n !}=\frac{u_{k, \lambda}\left(1-e_{\lambda}(-t) \mid p\right)}{t\left(1-y\left((1+\lambda t)^{\frac{1}{\lambda}}-1\right)\right)}(1+\lambda t)^{\frac{x}{\lambda}} \\
& =\left(\sum_{n=0}^{\infty} F_{n, \lambda, p}^{(k)} \frac{t^{n}}{n !}\right)\left(\sum_{m=0}^{\infty}\left(\begin{array}{c}
\frac{x}{\lambda} \\
m
\end{array}\right) \lambda^{m}(\log (1+z))^{m}\right) \\
& =\left(\sum_{n=0}^{\infty} F_{n, \lambda, p}^{(k)} \frac{z^{n}}{n !}\right)\left(\sum_{m=0}^{\infty}(x)_{m, \lambda} \sum_{l=m}^{\infty} S_{1}(l, m) \frac{z^{l}}{l !}\right) \\
& =\left(\sum_{n=0}^{\infty} F_{n, \lambda, p}^{(k)} \frac{z^{n}}{n !}\right)\left(\sum_{l=0}^{\infty}\left(\sum_{m=0}^{l}(x)_{m, \lambda} S_{1}(l, m)\right) \frac{z^{l}}{l !}\right) \\
& =\sum_{n=0}^{\infty}\left(\sum_{l=0}^{n} \sum_{m=0}^{l}\left(\begin{array}{l}
n \\
l
\end{array}\right)(x)_{m, \lambda} S_{1}(l, m) F_{n-l, \lambda, p}^{(k)}\right) \frac{z^{n}}{n !} .
\end{aligned}
$$

By equation (3.10), we get the following theorem.

Theorem 3.3. Let $j$ be nonnegative integer and $k \in \mathbb{Z}$. Then

\section{Conclusion}

$$
F_{n, \lambda, p}^{(k)}(x)=\sum_{l=0}^{n} \sum_{m=0}^{l}\left(\begin{array}{l}
n \\
l
\end{array}\right)(x)_{m, \lambda} S_{1}(l, m) F_{n-l, \lambda, p}^{(k)} .
$$

In this paper, we introduced the new type of degenerate poly-Fubini polynomials and numbers by using the degenerate polylogarithm function and derive several properties on the degenerate poly-Fubini numbers and polynomials. We represented the generating function of the degenerate poly-Fubini numbers by iterated integrals in Theorem 2.2, 2.3, and 2.4 and explicit degenerate poly-Euler polynomials in terms of the Bernoulli polynomials and degenerate Stirling numbers of the second kind in Theorem 2.5. We also represented those numbers in terms of the degenerate Stirling numbers of the second kind in Theorem 2.6. In the final section, we defined the degenerate unipoly-Fubini polynomials by using the degenerate polylogarithm function and obtained the identity degenerate unipoly-Fubini polynomials in terms of the degenerate Fubini numbers and degenerate Stirling numbers of the second kind in Theorem 3.1 and Theorem 3.2, the degenerate unipoly-Fubini numbers and the Stirling numbers of the first kind in Theorem 3.3.

Author Contributions: All authors contributed equally to the manuscript and typed, read, and approved final manuscript.

\section{Funding: None.}

Acknowledgements: None.

Conflict of Interest: The authors declare no conflict of interest. 


\section{References}

[1] Boyadzhiev, K. N. A series transformation formula and related polynomials. Int. J. Math. Math. Sci. 2005, 23,3849-3866.

[2] Carlitz, L. Degenerate Stirling, Bernoulli and Eulerian numbers. Utilitas Math. 1979, 15, 51-88.

[3] Carlitz, L. A degenerate Staudt-Clausen theorem, Arch. Math. (Basel) 1956, 7, 28-33.

[4] Dil, A.; Kurt, V. Investing geometric and exponential polynomials with EulerSeidel matrices. J. Integer Sequences. 2011, 14,1-12.

[5] Duran, U.; Acikgoz, M. Truncated Fubini polynomials. Mathematics. 2019, 7, 431; doi:10.3390/math7050431.

[6] Dolgy, D.V.; Jang, L.C.; A note on the polyexponential Genocchi polynomials and numbers. Symmetry. 2020, 12,1007; doi:10.3390/sym12061007.

[7] Guohui, C,; Li, C. Some identities involving the Fubini polynomials and Euler polynomials, Mathematics. 2018, 6,300; doi:10.3390/math6120300.

[8] Kaneko, M. Poly-Bernoulli numbers. J. Théor Nombres Bordeaux. 1997, $9(1), 221-228$.

[9] Khan, W.A. A new class of degenerate Frobenius-Euler-Hermite polynomials. Adv. Stud. Contemp. Math. (Kyungshang). 2018, 28(4), 567-576.

[10] Khan, W.A.; Nisar, K.S.; Duran, U.; Acikgoz, M.; Araci, S. Multifarious implicit summation formulae of Hermite-based poly-Daehee polynomials. Appl. Math. Inf. Sci. 2018, 12(2), 305-310.

[11] Khan, W.A.; Nisar, K.S.; Baleanu, D. A note on $(p, q)$-analogue type of Fubini numbers and polynomials. AIMS Math. 2020, 5(3), 2743-2757.

[12] Khan, W.A.; Sharma, S.K. A new class of Hermite-based higher order central Fubini polynomials. Int. J. Appl. Comput. Math. 2020, 87(6), 1-14.

[13] Khan, W.A.; Ayesha, K.; Khan, I.A. Degenerate polyexponential-Genocchi numbers and polynomials. J. Math. Comput. Sci. 2021, 22, 381-381.

[14] Kim, D.S.; Kim, T. Higher-order Bernoulli and poly-Bernoulli mixed type polynomials. Georgian Math. J. 2015, 22, 265-272.

[15] Kim, D.S.; Kim, T. A note on polyexponential and unipoly functions. Russ. J. Math. Phys. bf 2019, 26(1), 40-49.

[16] Kim, T. A note on degenerate Stirling polynomials of the second kind. Proc. Jangjeon Math. Soc. 2017, 20(3), 319-331.

[17] Kim, T.; Jang,L.-C.; Kim, D.S.; Kim, H.-Y. Some identities on type 2 degenerate Bernoulli polynomials of the second kind. Symmetry. 2020, 12(4), Article ID 510(2020).

[18] Kim, T.; Kim, D.S. Degenerate Laplace transform and degenerate gamma function. Russ. J. Math. Phys. 2017, 24(2), 241-248.

[19] Kim, T.; Khan, W.A.; Sharma, S. K.; Ghayasuddin, M. A note on parametric kinds of the degenerate poly-Bernoulli and poly-Genocchi polynomials. Symmetry. 2020, 12(4), Article ID 614.

[20] Kim, T.; Kim, D. S.; Dolgy, D. V.; Kwon, J.; Some identities on generalized degenerate Genocchi and Euler numbers. Informatica. 2020, 31(4), 42-51.

[21] Kim, D.S.; Kim, T. A note on type 2 Changhee and Daehee polynomials. arXiv:1809.05217v1 [math.NT] 14 Sep 2018.

[22] Kim, D.S.; Kim, T.; Kwon, H.-I.; Park, J.-W. Two variable higher-order Fubini polynomials. J. Korean Math. Soc. 2018, 55(4), 975-986.

[23] Kim, T.; Kim, D.S.; Jang, G.-W. A note on degenerate Fubini polynomials. Proc. Jangjeon Math. Soc. 2017, 20(4), 521-531. 
[24] Kim, D.S.; Jang, G.-W.; Kwon, H.-I.; Kim, T. Two variable higher-order degenerate Fubini polynomials. Proc. Jangjeon Math. Soc. 2018, 21(1), 5-22.

[25] Kim, T.; Kim, D.S.; Kwon, J.; Lee, H. Degenerate polyexpnential functions and type 2 degenerate poly-Bernoulli numbers and polynomials. Adv. Differ. Equ. 2020, 2020:168, 12pp.

[26] Kim, D.S.; Kim, T. A note on a new type of degenerate Bernoulli numbers. Russ. J. Math. Phys. 2020, 27(2), 227-235.

[27] Kim, T.; Kim, D.S.; Kwon, J.; Kim, H.Y. A note on degenerate Genocchi and poly-Genocchi numbers and polynomials. J. Ineq. Appl. 2020, 2020:110, 13 pp.

[28] Kim, T.; Kim, D.S. Degenerate polyexponential functions and degenerate Bell polynomials. J. Math. Anal. Appl. 2020, 487(2), 124017.

[29] Kargin, L. Some formulae for products of Fubini polynomials with applications. 2016, arXiv:1701.01023v1[math.CA] 23 Dec 2016.

[30] Kilar, N.; Simsek, Y. A new family of Fubini type numbers and polynomials associated with Apostol-Bernoulli numbers and polynomials. J. Korean Math. Soc. 2017, 54(2), 1605-1621.

[31] Kilar, N.; Simsek, Some relationships between Fubini type polynomials and other special numbers and polynomials. AIP Conference Proc. 2019, 2116(1),doi.org/10.1063

[32] Lewin, L. Polylogarithms and Associated Functions; With a Foreword by A.J. Vander Poorten; North-Holland Publishing Co.: Amsterdam, NY, USA, 1981.

[33] Lee, D.S.; Kim, H.-Y.; Jang, L.-C. Type 2 degenerate poly-Euler polynomials. Symmetry. 2020, 12, Article ID 1011.

[34] Sharma, S. K.; Khan, W. A.; Ryoo, C. S. A parametric kind of the degenerate Fubini numbers and polynomials. Mathematics. 2020, 8,405; doi:10.3390/math8030405.

[35] Sharma, S. K.; Khan, W. A.; Ryoo, C. S. A parametric kind of Fubini polynomials of a complex variable. Mathematics. 2020, 8,643; doi:10.3390/math8040643./1.5114093.

[36] Sharma, S. K.; Khan, W. A.; Araci, S.; Ahmed, S.S. New type of degenerate Daehee polynomials of the second kind. Adv. Differ. Equ. 2020, 2020:428, 14 pp.

[37] Tanny, S. M. On some numbers related to Bell numbers. Canad. Math. Bull. 1974, 17,733-738. 